ARTICLE

\title{
Identification and characterization of N9- methyltransferase involved in converting caffeine into non-stimulatory theacrine in tea
}

Yue-Hong Zhang1,2,3,6, Yi-Fang Li,2,3,6, Yongjin Wang 1,3,6, Li Tan¹,3, Zhi-Qin Cao 1,2,3, Chao Xie 1,2,3, Guo Xie ${ }^{4}$, Hai-Biao Gong1,2,3, Wan-Yang Sun1,2,3, Shu-Hua Ouyang1,2,3, Wen-Jun Duan²,3, Xiaoyun Lu1,3, Ke Ding (1) 1,3, Hiroshi Kurihara ${ }^{1,2,3}$, Dan Hu (i) ${ }^{1,2,3 凶}$, Zhi-Min Zhang (iD) ${ }^{1,3 凶}$, Ikuro Abe (D) ${ }^{5 凶}$ \& Rong-Rong He (D) ${ }^{1,2,3 凶}$

Caffeine is a major component of xanthine alkaloids and commonly consumed in many popular beverages. Due to its occasional side effects, reduction of caffeine in a natural way is of great importance and economic significance. Recent studies reveal that caffeine can be converted into non-stimulatory theacrine in the rare tea plant Camellia assamica var. kucha (Kucha), which involves oxidation at the C8 and methylation at the N9 positions of caffeine. However, the underlying molecular mechanism remains unclear. Here, we identify the theacrine synthase CkTcS from Kucha, which possesses novel N9-methyltransferase activity using 1,3,7-trimethyluric acid but not caffeine as a substrate, confirming that $\mathrm{C} 8$ oxidation takes place prior to N9-methylation. The crystal structure of the CkTcS complex reveals the key residues that are required for the N9-methylation, providing insights into how caffeine $\mathrm{N}$-methyltransferases in tea plants have evolved to catalyze regioselective $\mathrm{N}$-methylation through fine tuning of their active sites. These results may guide the future development of decaffeinated drinks.

\footnotetext{
${ }^{1}$ International Cooperative Laboratory of Traditional Chinese Medicine Modernization and Innovative Drug Development of Chinese Ministry of Education (MOE), College of Pharmacy, Jinan University, Guangzhou, China. ${ }^{2}$ Guangdong Engineering Research Center of Chinese Medicine \& Disease Susceptibility, College of Pharmacy, Jinan University, Guangzhou, China. ${ }^{3}$ Guangdong Province Key Laboratory of Pharmacodynamic Constituents of TCM and New Drugs Research, College of Pharmacy, Jinan University, Guangzhou, China. ${ }^{4}$ Zhongshan Institute, University of Electronic Science and Technology of China, 528402 Zhongshan, China. ${ }^{5}$ Graduate School of Pharmaceutical Sciences, The University of Tokyo, Tokyo 113-0033, Japan. ${ }^{6}$ These authors contributed equally:

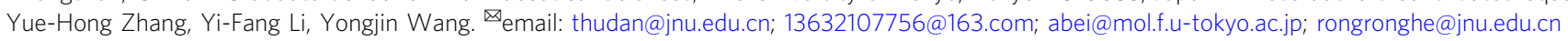


C affeine is well-known as a central nervous system stimulant and a major xanthine alkaloid component in many popular beverages, especially coffee (Coffea arabica and Coffea canephora) and tea (Camellia sinensis). Extensive studies have well established the main biosynthetic pathway of caffeine from xanthosine (1), which proceeds through 7-methylxanthine (2) and theobromine (3) to yield caffeine (4) (Fig. 1) (1,2 $^{1}$. At least three different $N$-methyltransferases are required to successively add methyl groups on the $N 7, N 3$, and $N 1$ - positions, with $S$-adenosyl- ${ }_{\mathrm{L}}$-methionine (SAM) as the methyl group donor ${ }^{3,4}$.

The pharmacological actions of caffeine are believed to be mediated via non-selectively antagonizing the $A_{1}$ and $A_{2 A}$ adenosine receptors in the central nervous system as well as peripheral tissues of the cardiovascular, respiratory, renal, and immune systems ${ }^{5}$. Consumption of caffeine may lead to occasional adverse effects, which include increased blood pressure, tremor, heart disease, anxiety, and gastrointestinal disturbances 6 . Awareness of these side effects has increased the demand for decaffeinated coffee and tea. However, producing a caffeine-free tea or coffee in a natural way that retains all the flavor and potential health benefit has proven to be challenging. A naturally decaffeinated arabica coffee species has been discovered, which has a mutatied caffeine synthase gene ${ }^{7}$. Alternatively, studies on the tea plant Camellia assamica var. kucha revealed that it removes caffeine in its leaves by converting caffeine into theacrine (6) (Fig. 1d) ${ }^{8}$.
Theacrine is a caffeine-like xanthine alkaloid with an additional methyl group at $N 9$ and a keto group at $C 8$. Despite its structural similarity to caffeine, theacrine does not cause side effects such as anxiety and dehydration. Instead, it has diverse beneficial biological activities, including anti-depressive ${ }^{9}$, sedative, and hypnotic activities $^{10,11}$, improving learning and memory ${ }^{12}$, increasing exercise activity ${ }^{13}$, and prevention of nonalcoholic fatty liver disease $^{14}$. Therefore, the large-scale production of transgenic theacrine-abundant plants could be an attractive proposition, but more information is needed about the enzymes involved in the biosynthesis of theacrine.

A previous study using ${ }^{14} \mathrm{C}$ labeled caffeine has confirmed that theacrine is biosynthesized from caffeine, possibly initiated by oxidation at $C 8$ to yield the intermediate 1,3,7-trimethyluric acid (5) followed by N9-methylation (Fig. 1d) ${ }^{8}$. However, the underlying molecular basis of this process remains unclear. Here, we identified three $\mathrm{N}$-methyltransferases involved in the biosynthesis of caffeine from the leaves of Kucha. Combined with enzymatic study and kinetic analysis, we found that one of the $\mathrm{N}$-methyltransferases, CkTcS, serves as the critical N9-methyltransferase, which only acts on 1,3,7-trimethyluric acid but not caffeine, indicating that $\mathrm{C} 8$-oxidation must take place prior to N9methylation. The structural characterization of $\mathrm{CkTcS}$ identified key residues around the substrate binding pocket that are critical for N9-methyltransferse activity. Taken together, these studies provide key mechanistic insights into theacrine biosynthesis,

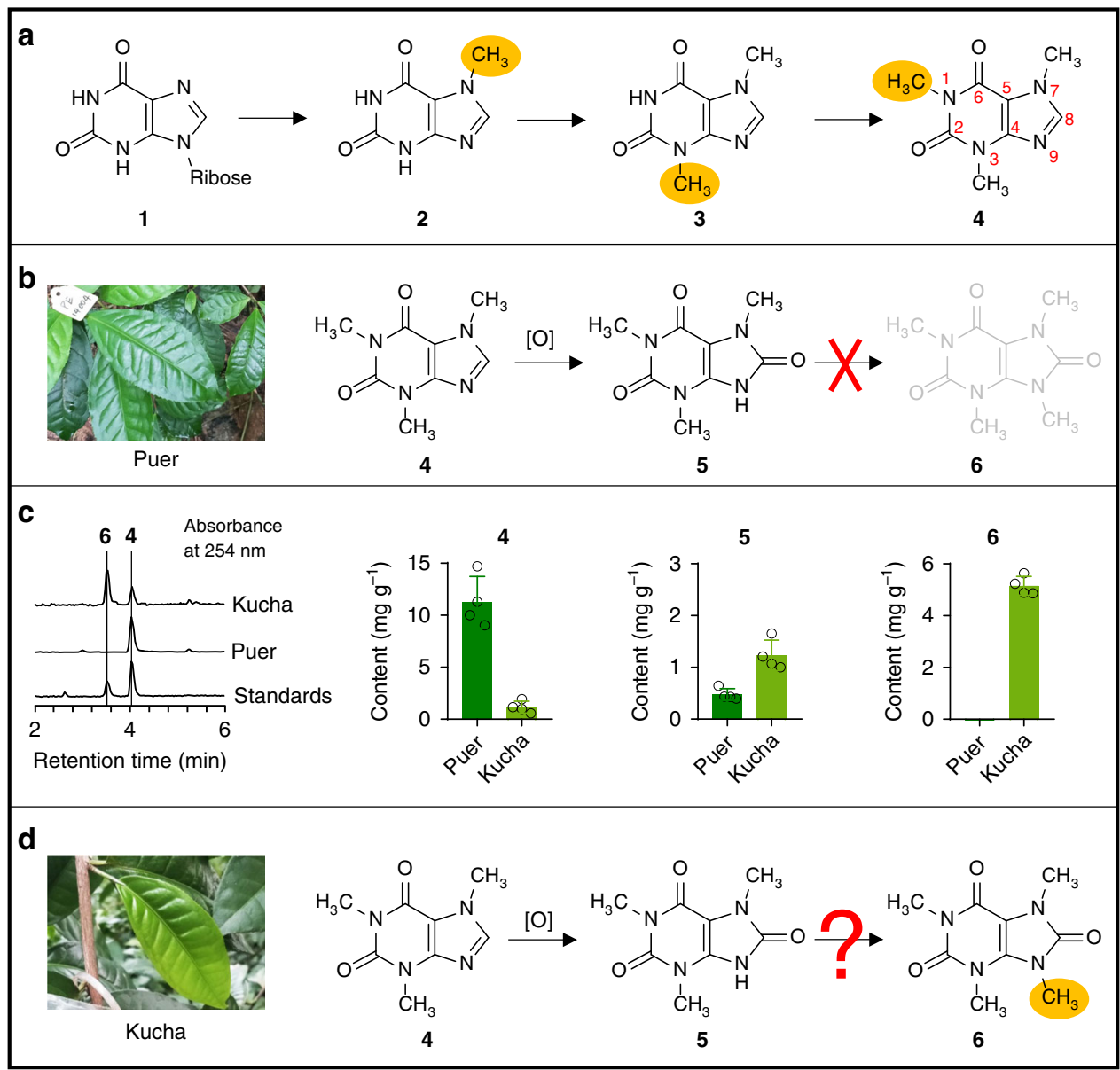

Fig. 1 Qualitative and quantitative analysis of major xanthine alkaloids in Puer and Kucha. a The main biosynthetic pathway of caffeine (4) from xanthosine (1). b Conversion of caffeine to 1,3,7-trimethyluric acid does not progress to theacrine in Puer leaves. c The content of caffeine (4), 1,3,7trimethyluric acid (5) and theacrine (6) in Puer and Kucha leaves. The left panel shows the HPLC analysis of $\mathbf{4}$ and $\mathbf{6}$ at absorbance wavelength of $254 \mathrm{~nm}$. The right three panels show the quantification of $\mathbf{4}, \mathbf{5}$, and $\mathbf{6}$ by HPLC-MS. Data represents mean \pm SD $(n=4)$. The corresponding dot plots are overlaid on the figure. d Conversion of caffeine to 1,3,7-trimethyluric acid then to theacrine in Kucha leaves. 
shedding light on a new direction for producing decaffeinated drinks.

\section{Results}

Cloning $\mathrm{N}$-methyltransferases from Kucha. To date, Kucha is the only plant reported to accumulate large quantities of theacrine. We used LC-MS analysis to compare the contents of caffeine (4), 1,3,7-trimethyluric acid (5), and theacrine (6) between Kucha and caffeine-enriched Puer tea (Camellia sinensis var. assamica) (Fig. 1c and Supplementary Fig. 1c). As expected, Kucha mainly contains theacrine and a small amount of caffeine, while Puer mainly contains caffeine without theacrine (Fig. 1c and Supplementary Fig. 1b). In addition, we detected a very small amount of caffeine metabolic intermediate 5 in both Kucha and Puer (Fig. 1c and Supplementary Fig. 1a). This result is consistent with the previous hypothesis that theacrine is synthesized from caffeine with 5 as an intermediate (Fig. 1d) ${ }^{8}$.

The existence of the intermediate $\mathbf{5}$ in both Kucha and Puer prompted us to hypothesize that a key N9-methyltransferase may exist in Kucha, but not in Puer (Fig. 1b, d). In order to clone the N9-methyltransferase involved in theacrine biosynthesis, we extracted the total RNA from both Kucha and Puer and performed transcriptome sequencing. Blast analysis of the transcriptome data using the amino acid sequence of tea caffeine synthase 1 (TCS1) (Accession No: AB031280), a well-characterized N1, N3methyltransferase in caffeine biosynthesis of tea, identified a partial $\mathrm{N}$-terminal sequence of an $\mathrm{N}$-methyltransferase (sequence ID: 35564), which is only expressed in Kucha, but not in Puer (Supplementary Fig. 2). Based on its sequence information, we designed primers to clone the potential N9-methyltransferase genes from the cDNA library derived from young Kucha leaves by PCR (Supplementary Fig. 3). As a result, three $N$-methyltransferases were cloned and named as $\mathrm{CkCS}, \mathrm{CkTbS}$, and $\mathrm{CkTcS}$, respectively.

Phylogenetic analysis of the three $N$-methyltransferases identified in Kucha with those characterized in tea and coffee reveals that $N$-methyltransferases from tea fall within a clade distinct from those of coffee (Supplementary Fig. 4a). This is consistent with the previous proposal that $N$-methyltransferases from tea and coffee are evolved from two independent origins via convergent evolution ${ }^{15-17}$. A focused view of the phylogenetic relationships of the $N$-methyltransferases identified from tea showed that CkCS and CkTbS group with N1/N3 and N3methyltransferases, respectively. In contrast, $\mathrm{CkTcS}$ is relatively distant to these known methyltransferases (Supplementary Fig. $4 \mathrm{~b}$ ), suggesting that it may possess a distinct function.

CkTcS is a N9-methyltransferase. To identify the N9-methyltransferase, we over-expressed all of them in Escherichia coli and purified the recombinant proteins to high purity (Fig. 2a). The enzymatic assay was performed using 1,3,7-trimethyluric acid (5) as a substrate, followed by HPLC analysis of the products. As shown in Fig. 2b, CkTcS exhibits a significant N9-methylation activity for converting 5 to theacrine (6), whereas only a miniscule amount of $\mathbf{6}$ was detected in the reactions of $\mathrm{CkCS}$ and CkTbS, suggesting that $\mathrm{CkTcS}$ is the target $N 9$-methyltransferase. Notably, we did not detect any product when using caffeine (4) as a substrate (Fig. 2d, Supplementary Fig. 5a, b), confirming that oxidation at $C 8$ of caffeine must take place prior to N9methylation.

To further verify the N9-methylation activity of $\mathrm{CkTcS}$, kinetic analysis of the three $N$-methyltransferases was performed. We initially measured the $K_{\mathrm{m}}$ parameter of $\mathrm{CkTcS}$ for SAM to determine the saturating SAM concentration. At the saturating concentration of $50 \mu \mathrm{M}$ substrate 5 , the $K_{\mathrm{m}}$ value for SAM is about $109.50 \mu \mathrm{M}$ (Supplementary Fig. 6a and Supplementary
Table 1). Based on this, a saturating SAM concentration of 1.5 $\mathrm{mM}$ was used in the subsequent kinetic experiments. Under these conditions, we measured the initial rates of the three methyltransferases at different substrate concentrations ranging from 0 to $2500 \mu \mathrm{M}$ (Fig. 2c). Fitting the initial rate data to the MichaelisMenten equation allowed us to derive kinetic parameters. Consistent with the enzymatic assay, CkCS and CkTbS show very weak N9-methyltransferase activity, with the $k_{\text {cat }} / K_{\mathrm{m}}$ value of $1.68 \mathrm{~s}^{-1} \mathrm{M}^{-1}$ and $1.11 \mathrm{~s}^{-1} \mathrm{M}^{-1}$, respectively. In contrast, CkTcS has a much higher $k_{\text {cat }} / K_{\mathrm{m}}$ value of $2440.17 \mathrm{~s}^{-1} \mathrm{M}^{-1}$, which is about 1500 times that of CkCS, and more than 2000 times that of CkTbS (Fig. 2c and Supplementary Table 1). These results indicate that $\mathrm{CkTcS}$ is a potent $\mathrm{N9}$-methyltransferase.

In addition, the activity of $\mathrm{CkTcS}$ as well as CkCS and CkTbS towards other substrates including xanthosine (1), 7-methylxanthine (2), and theobromine (3) were also investigated. $\mathrm{CkTcS}$ shows very weak N3 and N1 methylation activities (Fig. 2d). This was further confirmed by kinetic analysis (Supplementary Fig. $6 \mathrm{~b}$ and Supplementary Table 1) and time-course comparison (Supplementary Fig. 6c). On the other hand, CkCS and CkTbS exhibit notable N1/N3 (Supplementary Fig. 5a) and N3 (Supplementary Fig. 5b) methyltransferase activities, respectively, which is consistent with the phylogenetic analysis (Supplementary Fig. 4)

The accumulation of theacrine in Kucha but not in Puer was first thought to be caused by the deficiency of CkTcS gene in Puer. However, an $N$-methyltransferase gene with identical amino acid sequence to $\mathrm{CkTcS}$ was also cloned from Puer using the same primers, suggesting an alternative reason for theacrine accumulation in Kucha. We then compared the expression of CkTcS between Puer and Kucha. As a result, we found that the expression of CkTcS in Kucha was significantly higher than that in Puer (Fig. 3), suggesting that the significant expression of the $\mathrm{CkTcS}$ is the main reason for the accumulation of theacrine in Kucha.

Crystal structure of CkTcS. The structures of two N-methyltransferases catalyzing N7 (XMT) and N3, N1 (DXMT) methylation in Coffea canephora have been reported ${ }^{18}$, but there is still no structure of $\mathrm{N}$-methyltransferase derived from tea plants. To explain the substrate specificity of $\mathrm{CkTcS}$, we determined the crystal structure of $\mathrm{CkTcS}$ in complex with 1,3,7-trimethyluric acid (5), in the presence of $S$-adenosyl- ${ }_{\mathrm{L}}$-homocysteine (SAH), a byproduct of the cofactor SAM, at a resolution of $3.14 \AA$ (Fig. 4, Supplementary Fig. 7 and Supplementary Table 2). Like the coffee methyltransferases, $\mathrm{CkTcS}$ exists as a homodimer, and this dimeric arrangement is preserved in the crystal lattice, with the dimer interface composed predominantly of hydrophobic interactions in the core region surrounded by hydrogen bonding interactions. The structure of $\mathrm{CkTcS}$ complex adopts a similar fold, SAH and substrate binding pockets to that of XMT and DXMT, with a backbone root-mean-square deviation (RMSD) of 1.24-1.32 $\AA$ over $251 \mathrm{Ca}$ atoms (Fig. 4b). Each monomer of the CkTcS complex consists of a core SAM-dependent methyltransferase domain and a short $a$-helical cap domain, both of which are involved in the binding of SAH and $\mathbf{5}$ (Supplementary Fig. 7). The major structural difference between the coffee and CkTcS N-methyltransferases occurs on a helix (residues 236-255 in $\mathrm{CkTcS}$ ), which contributes to the formation of the substrate binding pocket. In $\mathrm{CkTcS}$, this helix is bent with the end close to the substrate moved away from the pocket (Fig. $4 \mathrm{~b}$ ).

The 1,3,7-trimethyluric acid binding pocket. The 1,3,7-trimethyluric acid is enveloped in the active site through both hydrogen bonding and van der Waals interactions, with $\mathrm{N}-9$ approximate to the thioether moiety of SAH (Supplementary 
a

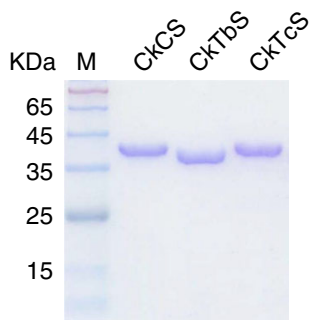

b 56 Absorbance

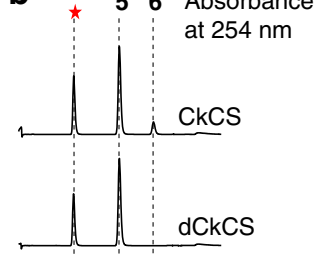

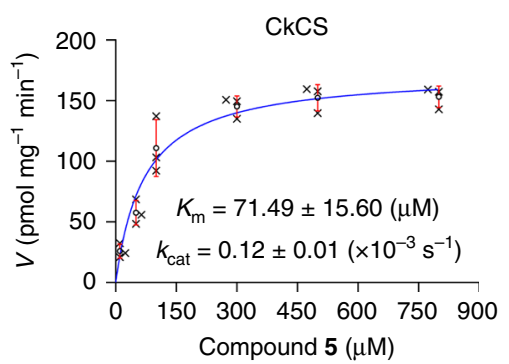
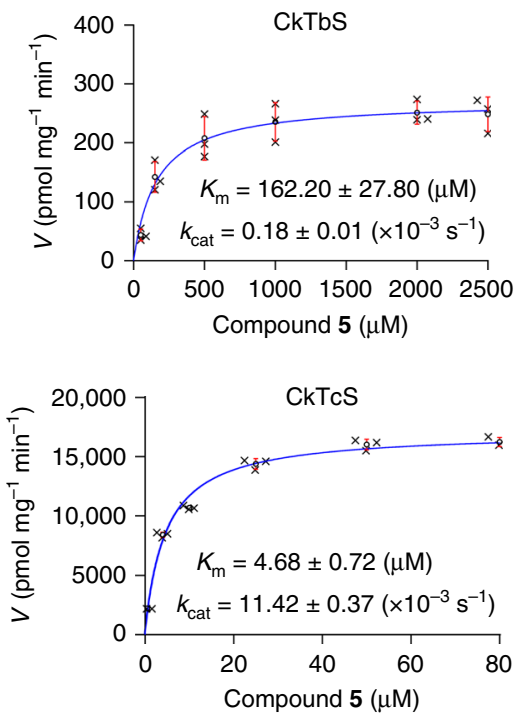

d
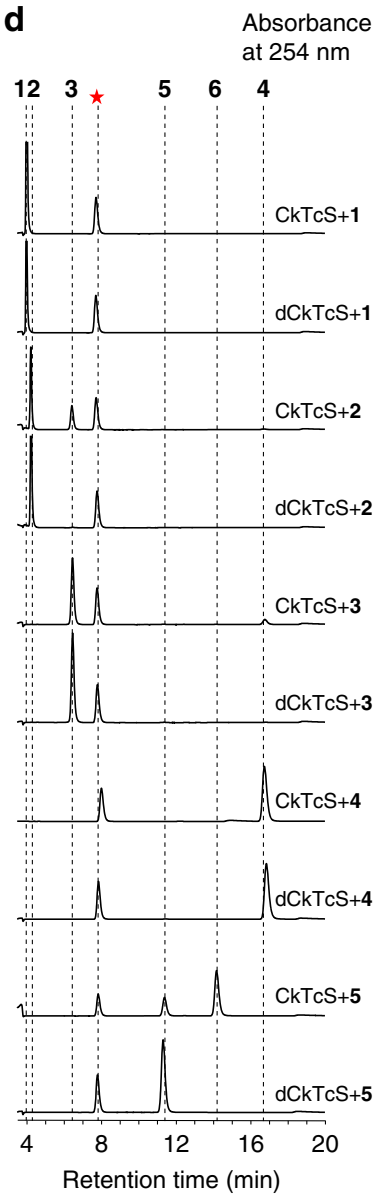

Fig. 2 Identification of N9-methyltransferase involved in converting caffeine into theacrine in tea leaves. a SDS-PAGE analysis of the recombinant $\mathrm{CkCS}, \mathrm{CkTbS}$, and CkTcS proteins. $\mathbf{b}$ In vitro N9-methyltransferase activity analysis of CkCS, CkTbS and CkTcS. HPLC analysis of in vitro reaction products using 1,3,7-trimethyluric acid (5) as a substrate with CkCS and denatured CkCS (dCkCS), CkTbS and denatured CkTbS (dCkTbS), CkTcS and denatured CkTcS (dCkTcS). The absorbance wavelength was set at $254 \mathrm{~nm}$. c Steady state kinetic analysis of CkCS, CkTbS, and CkTcS using 1,3,7-trimethyluric acid (5) as a substrate. Initial velocities are shown as cycles and represented as mean \pm SD $(n=3)$. The corresponding dot plots are overlaid on the figure. The blue line represents the nonlinear least-squares fit of the initial velocities versus 1,3,7-trimethyluric acid concentration to the hyperbolic Michaelis-Menten equation. Kinetic parameters were determined at a saturating concentration of $1.5 \mathrm{mM} \mathrm{SAM}$. $\mathbf{d}$ In vitro N-methyltransferase activity of CkTcS and dCkTcS towards xanthosine (1), 7-methylxanthine (2), theobromine (3), caffeine (4), and 1,3,7-trimethyluric acid (5). The absorbance wavelength was set at 254 $\mathrm{nm}$. In all HPLC chromatograms, a red asterisk indicates an impurity compound from the SAM reagent.

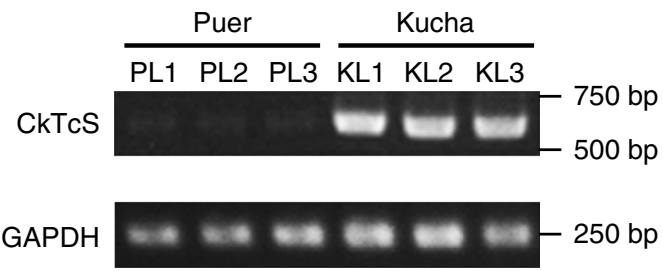

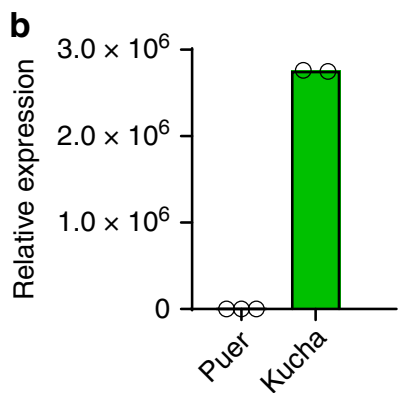

Fig. 3 Transcriptional level analysis of CkTcS gene in Kucha and Puer. a Qualitative analysis of CkTcS and GAPDH expression in Puer and Kucha. PL1, PL2, PL3, and KL1, KL2, KL3 represent three different leaves from Pure tea plant and Kucha tea plant, respectively. b Quantitative analysis of CkTcS and GAPDH expression in Puer and Kucha by quantitative real-time PCR. The gene expression in each leaf was analyzed at three replicates. The PCR was run for 40 cycles. Expression of CkTcS relative to GAPDH in three Pure leaves versus two Kucha leaves is shown.

Fig. 7). Residues surrounding the binding pocket include Met-15, Tyr-24 from the cap domain and Phe-30, Thr-31, Tyr-157, His160, Trp-161, Arg-226, Ile-241, Trp-242, Cys-270, Ile-318, and Phe-322 from the methyltransferase domain (Fig. 4c, d). Some of these positions are commonly used by tea $N$-methyltransferases, coffee $N$-methyltransferases and even far related salicylic acid carboxyl methyltransferases (SAMT) ${ }^{19}$, especially Met-15, Tyr24, Try-157, Trp-161, and Ile-318, which are sequentially or 
a

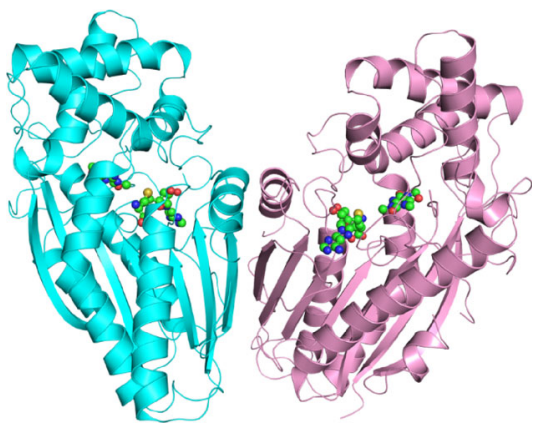

C

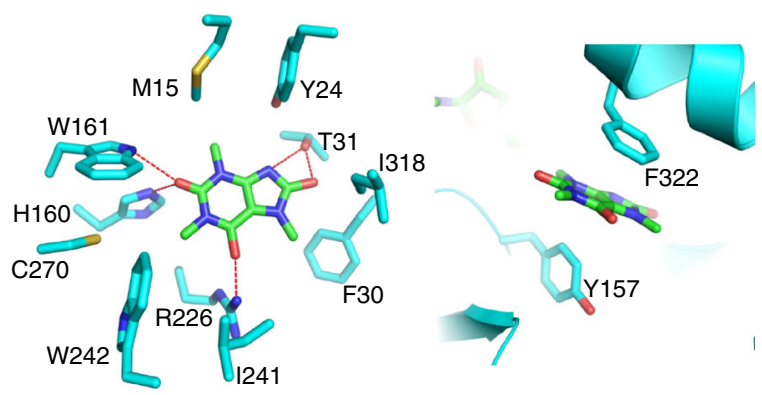

b

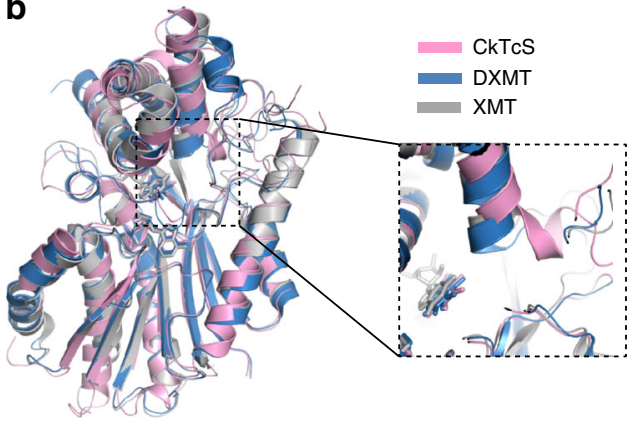

d

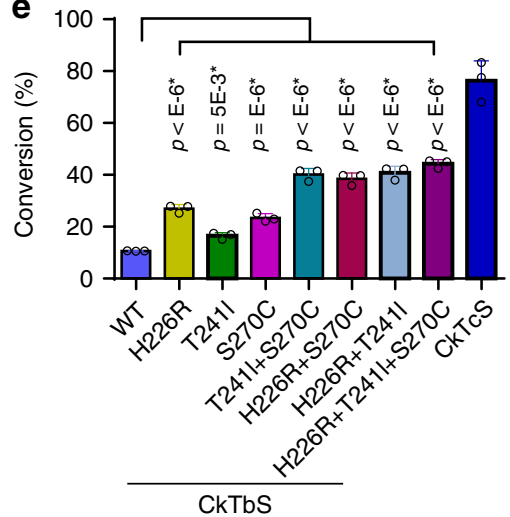

Fig. 4 Crystal structure of CkTcS. a Crystal structure of CkTcS dimer. SAH and 1,3,7-trimethyluric acid (5) are shown in ball-and-stick representation. b Structural overlap of CkTcS (pink) with DXMT (blue) and XMT (grey), with the major structural difference around the substrate binding pocket between CkTcS, DXMT, and XMT highlighted. c A close-up view of the CkTcS-1,3,7-trimethyluric acid interactions. The hydrogen bonds are shown in red dashed lines. $\mathbf{d}$ A close-up view of the $\pi$ - $\pi$-stacking interactions. e In vitro methylation assay of wild-type (WT) or mutants of CkTbS using 1,3,7-trimethyluric acid as substrate, with $\mathrm{CkTCS}$ as positive control. Data represent mean $\pm \mathrm{SD}(n=3)$. The corresponding dot plots are overlaid on the figure. The experiment was repeated twice. Differences were assessed statistically by two-tailed Student's $t$-test, ${ }^{\star \star \star \star} P<0.0001,{ }^{\star} P<0.05$.

functionally conserved (Supplementary Fig. 8). These residues may form the primary interactions modulating the threedimensional architecture of the substrate binding pocket.

In the $\mathrm{CkTcS}$ structure, $\mathbf{5}$ makes a total of five hydrogen bonds to the protein, with all the distances between the hydrogen-bondforming atoms less than $3.0 \AA$. The $O-6$ carbonyl group of 5 is recognized by the $\mathrm{N \varepsilon}$ groups of Arg-226 through hydrogenbonds; the side-chain of Trp-161 and His-160 form direct hydrogen bonds with the $O-2$ carbonyl group of 5 (Fig. 4c). Particularly, the hydroxyl group of Thr-31 forms hydrogen bonds with both $N-9$ and $O-8$. The $O-8$ position is the only distinction between caffeine and $\mathbf{5}$, and it is critical for $\mathbf{5}$ to serve as substrate of CkTcS. The Thr-31-O-8 hydrogen bond may stabilize the $O-8$ carbonyl group in an iminol tautomeric state, which could facilitate attack of SAM for N9-methylation. The O-8 carbonyl group also interacts with the side-chain of Ile-318 through van der Waals interaction (Fig. 4c). Additionally, Phe-322 and Tyr157 together sandwich the purine ring of 5 through a $\pi-\pi$ stacking interaction (Fig. 4d).

N9-methylation activity of CkTcS. We next asked why CkTcS prefers $\mathbf{5}$, given the fact that it shares a sequence identity $>90 \%$ with CkTbS and CkCS. We tried to solve the structure of CkTbS and CkCS in the presence of SAH and their corresponding substrates, but only crystallized CkTbS. Unfortunately, the N-terminal cap domain of CkTbS is disordered due to crystal packing. We finally solved the structure of CkTbS in apo form (Supplementary Fig. 9). However, when overlapping the CkTbS and $\mathrm{CkTcS}$ structures, most residues lining the substrate binding pockets are in the same orientation (Supplementary Fig. 9), suggesting that substrate binding does not induce large conformational changes in the methyltransferase domain. The substrate specificity of $\mathrm{CkTcS}$ proteins might be determined by minor variable residues which subtly tailor the chemical features of the core catalytic scaffold. We first analyzed the residues surrounding the $O-8$ position. Both Phe-30 and Thr-31 are well preserved, but Ile-318 in CkTcS is replaced by a Met-318. However, the conformation of Met-318 is flexible, and it could potentially contribute a similar van der Waals interaction (Supplementary Fig. 9b). These suggest that $\mathrm{CkTcS}$ did not evolve a specific mechanism to distinguish 5 from other molecules on this position, even though the $O-8$ mediated interactions are critical for N9-methylation.

Further observation identified three sequence-variable residues around the pocket: CkTcS_Arg-226 (CkTbS_His-226, CkCS_Arg225), CkTcS_Ile-241 (CkTbS_Thr-241, CkCS_Thr-240), and CkTcS_Cys-270 (CkTbS_Ser-270, CkCS_Ser-269) (Supplementary Figs. 8 and 9). Except for Arg-226, both Ile-241 and Cys-270 are not involved in direct interaction with $\mathbf{5}$, and their distances to 5 are about $4.6 \AA$. Particularly, of the three residues, two are different between $\mathrm{CkTcS}$ and CkCS, three between CkTcS and CkTbS. Our kinetic study has indicated that CkCS has a higher $k_{\text {cat }} / K_{\mathrm{m}}$ value compared to that of CkTbS (Fig. $2 \mathrm{c}$ and Supplementary Table 1). Consistently, CkTbS H226R mutation led to an apparent increase of N9-methylation activity towards 5 (Fig. 4e). Taken together, these results suggest that Arg-226 plays an active role in binding of 5 and positioning 5 properly for $\mathrm{N} 9$ methylation. However, Arg-226 alone is not enough: Ile-241 and Cys-270 are also indispensable. In CkTbS and CkCS, Ile-241 is 
replaced by a threonine, and Cys-270 by a serine, both of which contain hydrophilic side-chains that may introduce extra direct or water-mediated hydrogen-bond and influence substrate binding. Single mutation of T241I or C270S on CkTbS led to a moderate increase in reaction products, while the double and triple mutations further increased the $N$-methyltransferase activity to almost half of that of $\mathrm{CkTcS}$ (Fig. 4e). Additionally, the equivalent residues of Ile-241 and Cys-270 in the structures of DXMT (Ser237 and Ile-266) and XMT (Ala-238 and Vle-267) are also involved in substrate discrimination ${ }^{18}$. Taken together, these results indicated that the N9-methylation activity of $\mathrm{CkTcS}$ depends on two layers of interaction: the hydrogen bond between Thr-31 and the O-8 carbonyl group to stabilize an iminol tautomeric state, and the combinatorial effort of three side-chains to accurately position 5 .

Convergent evolution of tea and coffee $\mathrm{N}$-methyltransferases. Genomic sequencing studies have revealed a convergent evolution of $N$-methyltransferases from tea and coffee plants ${ }^{20}$. We found tea $N$-methyltransferases position substrates with some different strategies from coffee $\mathrm{N}$-methyltransferases. One striking difference is their aromatic ring recognition. In DXMT, the purine ring of theobromine is clamped through a hydrophobic- $\pi$-stacking interaction, with the perpendicular Tyr-157 forming a $\pi$-stacking interaction on one face, whereas Ile-332 makes a hydrophobic interaction on the opposite face (Supplementary Fig. 10). Similar interactions are also observed in the structure of XMT and even SAMT ${ }^{18,19}$. Sequence alignment indicates that the Ile-332 position is occupied by a conserved phenylalanine residue in tea plant $N$-methyltransferases, whereas it is either an isoleucine or a valine residue in other $N$-methyltransferases (Supplementary Fig. 8). This suggests that the $\pi$ - $\pi$-stacking interaction might be a unique way of substrate recognition by tea plant $N$-methyltransferases. Another obvious difference is that there are two sequence-conserved tyrosine residues in $N$-methyltransferases of coffee plants that are involved in constructing the pocket. In the structures of DXMT (Tyr-333 and Tyr-368) and XMT (Tyr-321 and Tyr-356), both of the two tyrosine residues are involved in direct interaction with the substrates through hydrophobic interaction or hydrogen-bond ${ }^{18}$, but their counterparts in tea $\mathrm{N}$-methyltransferases are usually serine or threonine residues (Supplementary Fig. 8). In CkTcS, those residues are too distant from 5 (Supplementary Fig. 10). These results clearly indicated that the caffeine $N$-methyltransferases from tea and coffee adopt a distinct substrate binding strategy even though they possess the same methylation activity, which provides further evidence for the convergent evolution of the $\mathrm{N}$-methyltransferases from tea and coffee in terms of their enzyme structure.

\section{Discussion}

$N$-methyltransferases play key roles in the pathway of caffeine biosynthesis. Extensive studies have well established that caffeine biosynthesis relies on methylation on the N7, N1, and N3 positions by corresponding $N$-methyltransferases. This study presents, to our best knowledge, the first N9-methyltransferase in caffeine metabolism, which will expand our understanding of this important protein family.

Genomic sequencing studies have revealed a convergent evolution of the $N$-methyltransferases among tea and coffee plants. Usually, the sequence variety between $N$-methyltransferases with different catalytic activities from the same origin is less apparent than that of $N$-methyltransferases with the same catalytic activities from different origins. This is evident even though $\mathrm{N}$ methyltransferases from both origins share a similar structural fold, substrate binding pocket and dimeric conformation. To recognize different substrates and achieve high methylation- position specificity, these enzymes subtly manipulate the orientation of the substrate through the combined effort of a set of residues lining the binding pocket.

Our results confirmed that the conversion of caffeine to theacrine happens with oxidation at the $C 8$ position first, followed by methylation at the $N 9$ position. So far, the enzyme responsible for C8 oxidation is still unclear. The detection of $\mathbf{5}$ in both Kucha and Puer suggests that this enzyme might be ubiquitous in the tea plant, and the N9-methylation is the key step to consume $\mathbf{5}$ and produce theacrine. Surprisingly, we also cloned the CkTcS gene from Puer, which produces no theacrine at all, but its expression level is strictly controlled.

Traditionally, the level of caffeine in the plants is decreased through the low activity of caffeine biosynthetic genes or the rapid degradation of caffeine $\mathrm{e}^{21}$. The identification of N9methyltransferase could guide mutagenesis work on existing functionally redundant $N$-methyltransferases in some tea plants to convert caffeine to theacrine, which has diverse beneficial biological activities but not the side effects of caffeine. In fact, theacrine $\mathrm{e}^{22}$ and 1,3,7-trimethyluric acid were also isolated from coffee (Supplementary Fig. 11), suggesting that a similar conversion of caffeine to theacrine may exist in some coffee plants, too $^{23}$. The greatest benefit of this strategy is that the whole caffeine biosynthesis pathway is still intact in the plants, which may help to keep the quality and aroma of the coffee beans. Our study therefore points a new direction for production of caffeinedeficient drinks.

\section{Methods}

Chemicals. $S$-adenosyl- ${ }_{L}-$ methionine $(S A M)$ was purchased from Sangon Biotech $S$-adenosyl- ${ }_{\mathrm{L}}$-homocysteine (SAH) was purchased from Sigma-Aldrich. Xanthosine, theobromine and caffeine were obtained from Wako Pure Chemical Industries, Ltd. 7-methylxanthine was purchased from Target Molecule. 1,3,7-trimethyluric acid and theacrine were supplied by Shanghai Better-In Pharmaceutical Technology Co., Ltd.

Plant materials. Young leaves from the tea plants Puer (Camellia sinensis var. assamica) and Kucha (Camellia assamica var. kucha) were collected at the experimental farm of the University of Electronic Science and Technology, Zhongshan City, Guangdong Province, China. Samples were collected in the Spring. The leaves were frozen in dry ice immediately after harvest and stored at $-80^{\circ} \mathrm{C}$.

Analysis of major Xanthine alkaloids of tea and coffee. Tea leaves were initially dried in an oven at $85^{\circ} \mathrm{C}$ for about $1.5 \mathrm{~h}$. The dried tea leaves and fresh coffee beans were ground into fine powder using a ceramic mortar. The resulting powders of tea leaves $(50 \mathrm{mg})$ and fresh coffee beans $(1.0 \mathrm{~g})$ were incubated in distilled water at $95^{\circ} \mathrm{C}$ for $40 \mathrm{~min}$, respectively. The precipitate was removed by centrifugation at $12,240 \times g$ for $10 \mathrm{~min}$ after cooling. The supernatant was filtered by a $0.22 \mu \mathrm{M}$ nylon filter before injection. Analysis was performed on a liquid chromatography tandem with mass spectrometry (LC/MS) system using a Dionex Ultimate 3000 HPLC system coupled on-line to a Q-Exactive Hybrid Quadrupole-Orbitrap Mass Spectrometer (Thermo Fisher Scientific, San Jose, CA) using an HSS T3 column $(2.1 \times 100 \mathrm{~mm}, 1.8 \mu \mathrm{m}$, Waters Acquity). The analysis was performed using gradient solvents (A) $0.1 \%$ formic acid in water $(0.1 \%, v / v)$ and (B) acetonitrile at a flow rate of $0.4 \mathrm{~mL} \mathrm{~min}^{-1}$. The gradient was as follows: $8 \% \mathrm{~B}$ at $0 \mathrm{~min}, 8 \% \mathrm{~B}$ at $1.0 \mathrm{~min}, 13 \% \mathrm{~B}$ at $1.5 \mathrm{~min}, 15 \% \mathrm{~B}$ at $4.5 \mathrm{~min}, 17 \% \mathrm{~B}$ at $6.0 \mathrm{~min}, 27 \% \mathrm{~B}$ at $8.0 \mathrm{~min}$, $60 \% \mathrm{~B}$ at $13.0 \mathrm{~min}, 80 \% \mathrm{~B}$ at $15.0 \mathrm{~min}, 80 \% \mathrm{~B}$ at $17.0 \mathrm{~min}$. The column was maintained at $40^{\circ} \mathrm{C}$. The wavelength was set at $254 \mathrm{~nm} .2 \mu \mathrm{L}$ of sample solvent was subjected to LC/MS. Analysis was performed in both negative and positive ion mode at a resolution of 70,000 for full MS scan and 17,500 for $\mathrm{MS}^{2}$ scan in datadependent mode. The scan range for MS analysis was $m / z 100-1500$ with a maximum injection time of $100 \mathrm{~ms}$ using 1 microscan. An isolation window of 1.0 Da was set for $\mathrm{MS}^{2}$ scans. Capillary spray voltage was set at $3.5 \mathrm{kV}$ and $-2.5 \mathrm{kV}$ in positive and negative ion modes, respectively. Capillary temperature was set at $320{ }^{\circ} \mathrm{C}$. The S-lens RF level was set to 60 . The standard solutions of caffeine (12.2-194.0 $\left.\mu \mathrm{g} \mathrm{mL}^{-1}\right), 1,3,7$-trimethyluric acid $\left(6.1-195.3 \mathrm{ng} \mathrm{mL}^{-1}\right)$, and theacrine $\left(28.0-896.0 \mu \mathrm{g} \mathrm{mL}^{-1}\right)$ were prepared to quantitate the content of xanthine alkaloids in tea plants. In addition, the standard solutions of 1,3,7-trimethyluric acid (6.6-105.0 $\left.\mathrm{ng} \mathrm{mL}^{-1}\right)$ and theacrine (14.0-224.0 $\mathrm{ng} \mathrm{mL}^{-1}$ ) were prepared to determinate the content of xanthion alkaloids in fresh coffee beans. 
RNA extraction and cDNA preparation. The tea leaves were broken into pieces and ground in liquid nitrogen with a mortar. Total RNA was extracted following the manufacturer's protocol of the RNeasy plant mini kit (QIAGEN). The first strand cDNA was prepared with a PrimeScript ${ }^{\text {tw }} 1$ st Strand cDNA Synthesis Kit (TaKaRa).

Transcriptome sequencing. Transcriptome sequencing in this study was performed at the Novogene Bioinformatics Institute (Novogene, Beijing, China). Briefly, mRNA was purified from the total RNA of tea leaves and used to construct sequencing libraries using a NEBNext ${ }^{\circledR}$ Ultra" RNA Library Prep Kit for Illumina ${ }^{\circledR}$ (NEB, USA) according to the manufacturer's recommendations. The index codes were added to attribute sequences to each sample. After the qualities of sequencing libraries were confirmed on the Agilent Bioanalyzer 2100 system, clustering of the index-coded samples was performed on a cBot Cluster Generation System using TruSeq PE Cluster Kit v3-cBot-HS (Illumia, San Diego, CA, USA) according to the manufacturer's instructions. The library preparations were then sequenced on an Illumina Hiseq 2500 platform (Illumina, San Diego, CA, USA) and paired-end reads were generated. Transcriptome assembly was accomplished based on the left. fq and right.fq using Trinity with min_kmer_cov set to 2 by default and all other parameters set default. Gene function was annotated based on the following databases: NCBI non-redundant Protein (NR), NCBI non-redundant nucleotide (NT), Protein family (Pfam), Clusters of Orthologous Groups of proteins (KOG/ COG), Swiss-prot, KEGG Ortholog (KO) and Gene Ontology (GO). Gene expression levels were estimated by RNA-Seq by Expectation Maximization (RSEM) for each sample.

Cloning of the $\boldsymbol{N}$-methyltransferase gene sequence. Transcriptome sequencing was completed by Beijing Nuohezhiyuan Technology Service Co, Ltd. A pair of oligonucleotide primers, $5^{\prime}$-ATGGAGCTAGCTACTAGGG- ${ }^{\prime}$ and $5^{\prime}$-CTATCCAACAATCTTGGAAAGC-3', were designed based on transcriptome data. The forward primer contains the start codon region of 35564 sequence and the reverse primer contains the stop codon region of 35562 sequence, respectively. Polymerase chain reaction (PCR) was performed using Prime STAR ${ }^{\circledast}$ HS DNA Polymerase (TaKaRa) in $50 \mu \mathrm{L}$ of reaction mixture containing cDNA and the primers mentioned above. The PCR protocol includes denaturation at $98^{\circ} \mathrm{C}$ for $2 \mathrm{~min}, 35$ cycles of denaturation at $98^{\circ} \mathrm{C}$ for $10 \mathrm{~s}$, annealing at different temperatures for $15 \mathrm{~s}$, and extension at $72{ }^{\circ} \mathrm{C}$ for $70 \mathrm{~s}$, followed by a final extension at $72{ }^{\circ} \mathrm{C}$ for $5 \mathrm{~min}$. The amplified DNA fragments were purified by polyacrylamide gel electrophoresis and were subcloned into the pBluescript II SK(+) vector. Subsequently, the resulting plasmids were transformed into Escherichia. coli (DH5a). Twenty clones were randomly selected in each transformation. DNA sequencing was performed by Shanghai Shenggong Bioengineering Co., Ltd. Nucleotide sequences were analyzed with the CLC sequence Viewer 7 program.

Protein purification. The cDNAs encoding CkCS, CkTbS and CkTcS were cloned into a pRSF-Duet vector with an $\mathrm{N}$-terminal $\mathrm{His}_{6}$-SUMO tag. The plasmids were verified by sequencing and then transformed into BL21(DE3) cells. When the cell density reached an $\mathrm{OD}_{600}$ of 0.6 , protein expression was induced using $0.4 \mathrm{mM}$ isopropyl $\beta$-D-1-thiogalactopyranoside (IPTG) at $16^{\circ} \mathrm{C}$ overnight. Cells were harvested, resuspended and lysed in buffer A [50 mM Tris- $\mathrm{HCl}(\mathrm{pH} 8.0), 25 \mathrm{mM}$ imidazole, $1 \mathrm{M} \mathrm{NaCl}$, and $1 \mathrm{mM}$ PMSF]. The recombinant proteins were purified using a Nickel column and eluted by buffer B $[25 \mathrm{mM}$ Tris- $\mathrm{HCl}(\mathrm{pH} 8.0), 250 \mathrm{mM}$ imidazole, $100 \mathrm{mM} \mathrm{NaCl}$. The eluted proteins were incubated with ubiquitin-likeprotease 1 (ULP1) on ice to cleave the $\mathrm{His}_{6}$-SUMO tag, and the tag-free proteins were further fractioned by ion-exchange (Q, GE Healthcare) and size-exclusion (Superdex 200, GE Healthcare) chromatography. Purified proteins were stored in a buffer containing $25 \mathrm{mM}$ Tris (pH 7.5), $100 \mathrm{mM} \mathrm{NaCl}, 5 \mathrm{mM}$ DTT at a concentration of $30 \mathrm{mg} \mathrm{mL}^{-1}$.

In vitro assay for $\mathbf{N}$-Methyltransferase. The $\mathrm{N}$-methylation in vitro assays were performed in triplicate at $27^{\circ} \mathrm{C}$ for $16 \mathrm{~h}^{24}$. Briefly, a $100 \mu \mathrm{L}$ reaction mixture contained $8 \mu \mathrm{M}$ purified recombinant protein (CkCS, CkTbS, and CkTcS), $1.5 \mathrm{mM}$ SAM, $400 \mu \mathrm{M}$ substrate $(\mathbf{1}, \mathbf{2}, \mathbf{3}, \mathbf{4}$, and 5), $100 \mathrm{mM}$ Tris- $\mathrm{HCl}(\mathrm{pH} 7.0)$, and $200 \mu \mathrm{M}$ $\mathrm{MgCl}_{2}$. The reactions were quenched by equal volumes of methanol. Boiled proteins were used as negative controls. The reaction mixture was centrifuged to remove precipitate, and then the supernatant was analyzed using the same LC system on a C18 reverse-phase column $(4.6 \times 250 \mathrm{~mm}, 5 \mu \mathrm{m}$, COSMOSIL). The analysis was performed using gradient solvents (A) $0.1 \%$ formic acid in water $(0.1 \%, \mathrm{v} / \mathrm{v})$ and $(\mathrm{B})$ acetonitrile at a flow rate of $1.0 \mathrm{~mL} \mathrm{~min}^{-1}$. The gradient was set as follows: $8 \% \mathrm{~B}$ at $0 \mathrm{~min}, 8 \% \mathrm{~B}$ at $5.0 \mathrm{~min}, 12 \% \mathrm{~B}$ at $20.0 \mathrm{~min}, 90 \% \mathrm{~B}$ at $20.1 \mathrm{~min}$, $90 \% \mathrm{~B}$ at $23.0 \mathrm{~min}$. The column temperature was maintained at $40^{\circ} \mathrm{C} .20 \mu \mathrm{L}$ of the supernatant was subjected to the HPLC-UV system. The wavelength was set at 254 $\mathrm{nm}$. Reaction products were identified by comparing retention times and ultraviolet spectra with those of authentic standards. The peaks of the substrates and products were integrated to calculate the conversion efficiency.

To compare the methylation activity of CkTcS between theobromine (3) and 1,3,7- trimethyluric acid (5), reactions were performed in a volume of $200 \mu \mathrm{L}$ containing $10 \mu \mathrm{M}$ purified protein $\mathrm{CkTcS}, 1.5 \mathrm{mM} \mathrm{SAM}, 500 \mu \mathrm{M}$ substrate ( 3 and 5), $100 \mathrm{mM}$ Tris- $\mathrm{HCl}(\mathrm{pH} 7.0)$ and $200 \mu \mathrm{M} \mathrm{MgCl}_{2}$. The mixtures were incubated at $27^{\circ} \mathrm{C}$ for varying times $(15,60,120,240,480 \mathrm{~min})$. After centrifugation, $20 \mu \mathrm{L}$ supernatant was subjected to HPLC-UV system and isocratically eluted at $8 \%$ B for $18 \mathrm{~min}$ using the same solvent system mentioned above.

Kinetic analysis. Compounds 3 and $\mathbf{6}$ were quantified by HPLC-MS and SAM was quantified by HPLC-UV. All were calculated using the external standard method with their corresponding calibration curves as shown in Supplementary Fig. 12.

The kinetic parameters of CkTcS with SAM were measured by consumption of SAM. The reactions were performed in a final volume of $200 \mu \mathrm{L}$ containing $1 \mu \mathrm{M}$ purified enzyme CkTcS, $100 \mathrm{mM}$ Tris- $\mathrm{HCl}$ (pH 7.0), $200 \mu \mathrm{M} \mathrm{MgCl}_{2}, 50 \mu \mathrm{M}$ compound 5 and varying concentrations $(10-600 \mu \mathrm{M})$ of SAM. The mixtures were incubated at $27^{\circ} \mathrm{C}$ for $3 \mathrm{~min}$ and then terminated by adding $200 \mu \mathrm{L}$ methanol. After centrifugation, $20 \mu \mathrm{L}$ of supernatant was subjected to HPLC-UV and was separated on a reverse-phase column $(4.6 \times 250 \mathrm{~mm}, 5 \mu \mathrm{m}$, COSMOSIL $)$ with the column temperature maintained at $40{ }^{\circ} \mathrm{C}$. The analysis was performed using a binary elution program with solvents (A) $0.1 \%$ formic acid in water $(0.1 \%, v / v)$ and (B) acetonitrile. The gradient was set as follows: $5 \% \mathrm{~A}$ at $0 \mathrm{~min}, 15 \% \mathrm{~A}$ at $3.5 \mathrm{~min}, 15 \% \mathrm{~A}$ at $7.0 \mathrm{~min}$, $5 \% \mathrm{~A}$ at $7.5 \mathrm{~min}, 5 \% \mathrm{~A}$ at $13.0 \mathrm{~min}$. The flow rate was set at $1.0 \mathrm{~mL} \mathrm{~min}{ }^{-1}$. The wavelength was set at $254 \mathrm{~nm}$. The same method was used to determine the kinetic parameters of other enzymes and substrates, with minor adjustments as follows:

The kinetic parameters of $\mathrm{CkCS}$ with $\mathbf{5}$ were measured by formation of product $\mathbf{6}$. $2 \mu \mathrm{M}$ CkCS was incubated with varying concentrations of substrate $5(10-800 \mu \mathrm{M})$ and $1.5 \mathrm{mM} \mathrm{SAM}$ for $10 \mathrm{~min}$. Product 6 was analyzed using the HPLC-MS system.

The kinetic parameters of CkTbS with $\mathbf{5}$ were measured by formation of product $6.4 \mu \mathrm{M}$ CkTbS was incubated with varying concentrations of substrate 5 $(50-2500 \mu \mathrm{M})$ and $1.5 \mathrm{mM}$ SAM for $15 \mathrm{~min}$. Product 6 was analyzed using HPLCMS system.

The kinetic parameters of $\mathrm{CkTcS}$ with 5 were measured by formation of product 6 . $0.2 \mu \mathrm{M}$ CkTcS was incubated with varying concentrations substrate $5(0.5-80 \mu \mathrm{M})$ and $1.5 \mathrm{mM}$ SAM for 3 min. Product 6 was analyzed using HPLC-MS system.

The kinetic parameters of $\mathrm{CkTcS}$ with 2 were measured by formation of product 3 . $1 \mu \mathrm{M}$ CkTcS was incubated with varying concentrations substrate $2(50-2500 \mu \mathrm{M})$ and $1.5 \mathrm{mM}$ SAM for $15 \mathrm{~min}$. Product $\mathbf{3}$ was analyzed by the same HPLC-MS system as mentioned above with an isocratic elution at $8 \% \mathrm{~B}$ for $13 \mathrm{~min}$.

The Km and Vmax values were calculated using the Michaelis-Menten kinetics equation by nonlinear regression analysis with GraphPad Prism 6 (GraphPad Software, La Jolla, CA). Kinetics were performed in triplicate and each data point represents the mean of three independent assays with error bars representing the standard deviation $( \pm \mathrm{SD})$.

Transcript level analysis of CkTcS gene in Puer and Kucha. RNAs from Kucha and Puer leaves were extracted using a RNeasy plant mini kit (Qiagen), and $2 \mu \mathrm{g}$ of RNA was reverse transcribed to cDNA using TransScript $^{\oplus}$ One-Step gDNA Removal and cDNA Synthesis SuperMix. Polymerase chain reaction (PCR) was performed using Prime STAR ${ }^{\oplus}$ HS DNA Polymerase (TaKaRa) in $50 \mu \mathrm{L}$ of reaction mixture containing cDNA and the gene-specific primers, $5^{\prime}$-TTCAAGCTATTAA CGCAGCA- $3^{\prime}$ and $5^{\prime}$-GGCTATAGCTAATAGTTCCCAAA- $3^{\prime}$, under the conditions of $98^{\circ} \mathrm{C}$ for $2 \mathrm{~min}, 28$ cycles of denaturation at $98^{\circ} \mathrm{C}$ for $10 \mathrm{~s}$, annealing at $55^{\circ} \mathrm{C}$ for $15 \mathrm{~s}$, and extension at $72{ }^{\circ} \mathrm{C}$ for $70 \mathrm{~s}$, followed by a final extension at $72{ }^{\circ} \mathrm{C}$ for 5 min. The PCR reaction conditions of the internal reference gene GAPDH (primers: $5^{\prime}$-ATCGTTGAGGGTCTCATGAC- $3^{\prime}$ and $5^{\prime}$-CACTGAGACATCGAC

AGTGG-3') were $98^{\circ} \mathrm{C}$ for $2 \mathrm{~min}, 35$ cycles of denaturation at $98^{\circ} \mathrm{C}$ for $10 \mathrm{~s}$, annealing at $50{ }^{\circ} \mathrm{C}$ for $15 \mathrm{~s}$, and extension at $72^{\circ} \mathrm{C}$ for $70 \mathrm{~s}$, followed by a final extension at $72^{\circ} \mathrm{C}$ for $5 \mathrm{~min}$. The amplified CkTcS and GAPDH gene was detected by polyacrylamide gel electrophoresis. In addition, the cDNA samples were amplified on a CFXConnect" Real-Time system (Bio-Rad, Hercules, CA, USA) in the presence of SYBR qPCR Master Mix (TOYOBO, OSAKA, JP) and specific primers described above. The mRNA expression of the CkTcS gene was calculated by the delta cycle threshold method, and the results were normalized to the reference gene GAPDH.

Crystallization and structure determination. For crystallization, eight residues on the $N$-terminal of $\mathrm{CkTcS}$ were truncated based on predicted structural disorder. CkTcS was mixed with $2 \mathrm{mM} \mathrm{SAH}$ and $5 \mathrm{mM}$ 1,3,7-trimethyluric acid on ice for 30 min to form a complex. The crystals were generated by the hanging-drop diffusion method at $4{ }^{\circ} \mathrm{C}$, from drops composed of $2 \mu \mathrm{L}$ of complex solution and $2 \mu \mathrm{L}$ of precipitant solution [0.1 M HEPES ( $\mathrm{pH}$ 7.5), $1 \mathrm{M}$ Sodium Citrate]. The full-length CkTbS was crystallized similarly in $0.1 \mathrm{M}$ MES (pH 6.0), $0.2 \mathrm{M} \mathrm{Li}_{2} \mathrm{SO}_{4}, 20 \%$ PEG4000. Glycerol was added to the precipitant solution to a final concentration of $15-20 \%(\mathrm{v} / \mathrm{v})$ to cryo-protect the crystals during flash freezing in liquid nitrogen X-ray diffraction data were collected on the beam-line BL17U1 at Shanghai Synchrotron Radiation Facility. The data were indexed, integrated and scaled by the HKL2000 package ${ }^{25}$. The structures were solved by molecular replacement using PHASER $^{26}$ and the DXMT structure (PDBcode: 2EFJ) as the searching model. Iterative cycles of model rebuilding and refinement were carried out using COOT (Version 0.8.1) ${ }^{27}$ and PHENIX (Version 1.9-1692) ${ }^{28}$, respectively. 1,3,7-trimethyluric acid and SAH were not filled into the electron densities until the proteins were well refined. The shape of electron density in the substrate binding pocket is consistent with the structure of 1,3,7-trimethyluric acid. Potential interactions of 1,3,7-trimethyluric acid with surrounding residues were also taken into 
consideration to determine its orientation. Data collection and refinement statistics are summarized in Supplementary Table 2.

Reporting summary. Further information on research design is available in the Nature Research Reporting Summary linked to this article.

\section{Data availability}

The structures of CkTcS-SAH-1,3,7-trimethyluric acid and CkTbS have been deposited in the Protein Data Bank under codes 6LYH and 6LYI, respectively. The GenBank accession numbers for nucleotide sequences of CkCS, CkTbS, and CkTcS are MN163829, MN163830, and MN163831, respectively. Assembled transcriptome data are available via DRYAD at https://datadryad.org/stash/share/ZymTdk5n80wG4-H7JebWmqJFkkkIBKn PqCPzJ1xE0y0. Additional data underlying Figs. 1c, 2c, 3b, 4e and Supplementary Figures 6 and 11 are available as a separate Source Data file.

Received: 1 October 2019; Accepted: 4 March 2020;

Published online: 19 March 2020

\section{References}

1. Negishi, O., Ozawa, T. \& Imagawa, H. Conversion of xanthosine into caffeine in tea plants. Agric. Biol. Chem. 49, 251-253 (1985).

2. Ashihara, H., Monteiro, A. M., Gillies, F. M. \& Crozier, A. Biosynthesis of caffeine in leaves of coffee. Plant Physiol. 111, 747-753 (1996).

3. Suzuki, T. \& Takahashi, E. Biosynthesis of caffeine by tea-leaf extracts. Enzymic formation of theobromine from 7-methylxanthine and of caffeine from theobromine. Biochem. J. 146, 87-96 (1975).

4. Suzuki, T. The participation of $S$-adenosylmethionine in the biosynthesis of caffeine in the tea plant. FEBS Lett. 24, 18-20 (1972).

5. López-Cruz, L., Salamone, J. D. \& Correa, M. Caffeine and selective adenosine receptor antagonists as new therapeutic tools for the motivational symptoms of depression. Front. Pharm. 9, 526 (2018).

6. Kato, M., Mizuno, K., Crozier, A., Fujimura, T. \& Ashihara, H. Caffeine synthase gene from tea leaves. Nature 406, 956-957 (2000).

7. Silvarolla, M. B., Mazzafera, P. \& Fazuoli, L. C. A naturally decaffeinated arabica coffee. Nature 429, 826 (2004).

8. Zheng, X.-Q., Ye, C.-X., Kato, M., Crozier, A. \& Ashihara, H. Theacrine (1,3,7,9-tetramethyluric acid) synthesis in leaves of a Chinese tea, kucha (Camellia assamica var. kucha). Phytochemistry 60, 129-134 (2002).

9. Guo, X. et al. Experimental study of theacrine on antidepressant effects. Chin. Pharmacol. Bull. 25, 1160-1163 (2009).

10. Xu, J.-K., Kurihara, H., Zhao, L. \& Yao, X.-S. Theacrine, a special purine alkaloid with sedative and hypnotic properties from Cammelia assamica var. kucha in mice. J. Asian Nat. Prod. Res. 9, 665-672 (2007).

11. Qiao, H. et al. Theacrine: a purine alkaloid from Camellia assamica var. kucha with a hypnotic property via the adenosine system. Neurosci. Lett. 659, 48-53 (2017).

12. Li, Y.-F. et al. Theacrine, a purine alkaloid derived from Camellia assamica var. kucha, ameliorates impairments in learning and memory caused by restraint-induced central fatigue. J. Funct. Foods 16, 472-483 (2015).

13. Feduccia, A. A. et al. Locomotor activation by theacrine, a purine alkaloid structurally similar to caffeine: involvement of adenosine and dopamine receptors. Pharmacol. Biochem. Be. 102, 241-248 (2012).

14. Wang, G.-E. et al. Theacrine protects against nonalcoholic fatty liver disease by regulating acylcarnitine metabolism. Metabolism 85, 227-239 (2018).

15. Xia, E.-H. et al. The tea tree genome provides insights into tea flavor and independent evolution of caffeine biosynthesis. Mol. Plant 10, 866-877 (2017).

16. Pichersky, E. \& Lewinsohn, E. Convergent evolution in plant specialized metabolism. Annu. Rev. Plant. Biol. 62, 549-566 (2011).

17. Denoeud, F. et al. The coffee genome provides insight into the convergent evolution of caffeine biosynthesis. Science 345, 1181-1184 (2014).

18. McCarthy, A. A. \& McCarthy, J. G. The structure of two $N$-methyltransferases from the caffeine biosynthetic pathway. Plant Physiol. 144, 879-889 (2007).

19. Zubieta, C. et al. Structural basis for substrate recognition in the salicylic acid carboxyl methyltransferase family. Plant Cell 15, 1704-1716 (2003).

20. Huang, R., O’Donnell, A. J., Barboline, J. J. \& Barkman, T. J. Convergent evolution of caffeine in plants by co-option of exapted ancestral enzymes. Proc. Natl Acad. Sci. USA 113, 10613-10618 (2016).

21. Nagai, C. et al. Production of a new low-caffeine hybrid coffee and the biochemical mechanism of low caffeine accumulation. Euphytica 164 133-142 (2008).

22. Petermann, J. B. \& Baumann, T. W. Metabolic relations between methylxanthines and methyluric acids in Coffea L. Plant Physiol. 73, 961-964 (1983).
23. Ashihara, H., Mizuno, K., Yokota, T. \& Crozier, A. Xanthine alkaloids: occurrence, biosynthesis, and function in plants. Prog. Chem. Org. Nat. Pro 105, 1-88 (2017).

24. Uefuji, H., Ogita, S., Yamaguchi, Y., Koizumi, N. \& Sano, H. Molecular cloning and functional characterization of three distinct $N$-methyltransferases involved in the caffeine biosynthetic pathway in coffee plants. Plant Physiol. 132, 372-380 (2003).

25. Otwinowski, Z. \& Minor, W. Processing of X-ray diffraction data collected in oscillation mode. Method Enzymol. 276, 307-326 (1997)

26. McCoy, A. J. et al. Phaser crystallographic software. J. Appl. Crystallogr. 40, 658-674 (2007).

27. Emsley, P. \& Cowtan, K. Coot: model-building tools for molecular graphics. Acta Crystallogr. D. Biol. Crystallogr. 60, 2126-2032 (2004).

28. Adams, P. D. et al. The Phenix software for automated determination of macromolecular structures. Methods 55, 94-106 (2011).

\section{Acknowledgements}

We thank Dr Wei-Xi Li and Ms Xing-Qian Sun for providing the coffee materials and photos. This work was supported, in part, by National Key Research and Development Program of China (grant number 2017YFC1700404), Natural Science Foundation of China (grant numbers 81622050, 81573675, U1801284, 81673709, 81873209, 31800638 and 31870032), the Local Innovative and Research Teams Project of Guangdong Pearl River Talents Program (grant number 2017BT01Y036) and GDUPS(2019), the Guangdong Natural Science Funds for Distinguished Young Scholar (2019B151502014), Science and Technology Program of Guangzhou (grant number 201903010062), and Natural Science Foundation of Guangdong Province (grant number 2018A030313003 and 2017A030306004). Assistance with Scientific English was provided by Dr. L.J. Sparvero (University of Pittsburgh)

\section{Author contributions}

R.R.H. and H.K. conceived and oversaw the project. I.A., D.H., and Z.M.Z. designed and managed the experiments. Y.H.Z. performed most of the experiments. Z.M.Z., Y.W., and L.T. constructed wild and mutant plasmids and purified proteins, performed crystallization trials, collected diffraction data, determined, and refined the structures. Z.Q.C. assisted with gene cloning and in vitro enzyme reaction experiment. C.X. assisted with vitro enzyme activity test analysis. C.X., G.X., and S.H.O. assisted with tea sample collection. W.Y.S., H.B.G., and S.H.O. assisted with qualitative and quantitative analysis of alkaloids in tea. W.J.D., assisted with the quantitative analysis of CkTcS gene expression in tea. X.L and K.D were involved in discussion of the results. Y.H.Z. and Y.F.L prepared the initial manuscript. D.H., Z.M.Z., I.A., and R.R.H. wrote the manuscript.

\section{Competing interests}

The authors declare no competing interests.

\section{Additional information}

Supplementary information is available for this paper at https://doi.org/10.1038/s41467020-15324-7.

Correspondence and requests for materials should be addressed to D.H., Z.-M.Z., I.A. or R.-R.H.

Peer review information Nature Communications thanks Andrew McCarthy and Joerg Ziegler for their contribution to the peer review of this work. Peer reviewer reports are available.

Reprints and permission information is available at http://www.nature.com/reprint

Publisher's note Springer Nature remains neutral with regard to jurisdictional claims in published maps and institutional affiliations.

Open Access This article is licensed under a Creative Commons Attribution 4.0 International License, which permits use, sharing, adaptation, distribution and reproduction in any medium or format, as long as you give appropriate credit to the original author(s) and the source, provide a link to the Creative Commons license, and indicate if changes were made. The images or other third party material in this article are included in the article's Creative Commons license, unles indicated otherwise in a credit line to the material. If material is not included in the article's Creative Commons license and your intended use is not permitted by statutory regulation or exceeds the permitted use, you will need to obtain permission directly from the copyright holder. To view a copy of this license, visit http://creativecommons.org/ licenses/by/4.0/.

(c) The Author(s) 2020 